\section{OPEN ACCESS}

Edited by:

George Elias Sarris,

Athens Heart Surgery Institute, Greece

Reviewed by:

Vladimiro Vida,

University of Padova, Italy Branko Mimic,

University Hospitals of Leicester United Kingdom

*Correspondence:

Camilla Sandrin

sandrini.camilla@gmail.com

Specialty section:

This article was submitted to

Pediatric Cardiology,

a section of the journal

Frontiers in Pediatrics

Received: 12 September 2019 Accepted: 27 December 2019

Published: 21 January 2020

Citation:

Sandrini C, Lombardi C, Shearn AIU,

Ordonez MV, Caputo M, Presti F, Luciani GB, Rossetti L and Biglino G (2020) Three-Dimensional Printing of

Fetal Models of Congenital Heart

Disease Derived From Microfocus

Computed Tomography: A Case

Series. Front. Pediatr. 7:567.

doi: 10.3389/fped.2019.00567

\title{
Three-Dimensional Printing of Fetal Models of Congenital Heart Disease Derived From Microfocus Computed Tomography: A Case Series
}

\section{Camilla Sandrini ${ }^{1 *}$, Claudio Lombardi ${ }^{2}$, Andrew I. U. Shearn ${ }^{3}$, Maria Victoria Ordonez ${ }^{3}$, Massimo Caputo ${ }^{3}$, Francesca Presti ${ }^{4}$, Giovanni Battista Luciani ${ }^{5}$, Lucia Rossetti ${ }^{1}$ and Giovanni Biglino ${ }^{3,6}$}

${ }^{1}$ Division of Cardiology, Department of Medicine, University of Verona, Verona, Italy, ${ }^{2}$ Studio Diagnostico Eco, Vimercate, Italy, ${ }^{3}$ Bristol Medical School, Bristol Heart Institute, University of Bristol, Bristol, United Kingdom, ${ }^{4}$ Division of Obstetrics and Gynecology B, Department of Surgery, Dentistry, Pediatrics and Gynecology, University of Verona, Verona, Italy, ${ }^{5}$ Division of Cardiac Surgery, Department of Surgery, Dentistry, Pediatrics and Gynecology, University of Verona, Verona, Italy, ${ }^{6}$ National Heart and Lung Institute, Imperial College London, London, United Kingdom

This article presents a case series of $n=21$ models of fetal cardiovascular anatomies obtained from post mortem microfocus computed tomography (micro-CT) data. The case series includes a broad range of diagnoses (e.g., tetralogy of Fallot, hypoplastic left heart syndrome, dextrocardia, double outlet right ventricle, atrio-ventricular septal defect) and cases also had a range of associated extra-cardiac malformations (e.g., VACTERL syndrome, central nervous system anomalies, renal anomalies). All cases were successfully reconstructed from the microfocus computed tomography data, demonstrating the feasibility of the technique and of the protocols, including in-house printing with a desktop 3D printer (Form2, Formlabs). All models were printed in 1:1 scale as well as with the 5-fold magnification, to provide insight into the intra-cardiac structures. Possible uses of the models include education and training.

Keywords: 3D printing, microfocus computed tomography, fetal heart, congenital heart disease, fetal cardiology, prenatal diagnosis

\section{INTRODUCTION}

Congenital heart defects are the most frequent congenital malformations. Advances in prenatal medicine allow early diagnosis, starting from the eleventh week of gestation $(1,2)$. Confirming the cardiac malformation after termination of pregnancy is essential for medical knowledge and familial counseling. Conventional autopsy is the gold standard technique for post mortem confirmation of congenital heart disease (CHD) but it lacks diagnostic power especially in challenging samples, such as cases of termination of pregnancy and of low dimension/weight $(3,4)$. In recent years, radiologic tools are becoming valid alternatives to conventional autopsies, in light of their non-destructive approach and high-resolution imaging capabilities (5-12). By using X-ray source on contrast-enhanced samples, post mortem microfocus computed tomography (microCT) can reach a spatial resolution of voxel sizes below 1 micron (i.e., $<0.001 \mathrm{~mm}$ ), thus allowing the evaluation of cardiac structures also in challenging specimens (5-9). Iodine preparation of the sample is necessary in order to create contrast between different cardiac soft tissues, that are otherwise not distinguishable (6). 
Three-dimensional (3D) printing in CHD is gaining increasing interest in the clinical and bioengineering communities alike, considering the breath of its applications. These include medical education, surgical, and catheter-based procedural individualized planning, and manufacturing research for device innovation (13-16). At present, applications of 3D printing in fetal cardiology are limited, yet the technique is appealing as precise visualization of small structures is essential to achieve a correct diagnosis, even in a very early in pregnancy. Pioneering work coming from in utero 3D echocardiography/magnetic resonance imaging (MRI) $(17,18)$ and from post mortem micro-CT (19) have been recently published.

Based on these early experiences, here we present a feasibility study of $3 \mathrm{D}$ printing normal fetal hearts and fetal hearts with CHD from micro-CT datasets, resulting in a case series of different diagnoses. The focus of the article is on technical issues of the imaging and printing processes.

\section{MATERIALS AND METHODS}

\section{Selection of Cases}

Post mortem micro-CT followed by conventional autopsy is offered to all patients referred to our Center for fetal echocardiography who decided for termination of pregnancy (below $21+6$ week of gestation, according to Italian Law) for either fetal cardiac anomalies or extra-cardiac malformations. Samples are managed according to previous described procedure (20). Post mortem micro-CT acquisitions are performed using a micro-CT SkyScan 1176 scanner (Bruker, Kontich, Belgio). Here we present the experience of consecutive cases collected between July 2016 and December 2018.

\section{Micro-CT Imaging Protocol}

Before performing micro-CT scans, the specimens were stained by immersion in Lugol solution 15\% (10 g potassium iodide and $5 \mathrm{~g}$ iodine in $100 \mathrm{ml}$ water) for $24 \mathrm{~h}$. Subsequently, the heart was washed with alcohol to remove free iodine, blotted dry, and scanned All scans were performed with a Bruker Skyscan 1176 micro-CT scanner (Bruker, Brussels Kontich, Belgium) with a gantry diameter of $68 \mathrm{~mm}$. Fixed acquisition times of $25 \mathrm{~min}$ were used for resolutions at $9 \mu \mathrm{m}$ and $6 \mathrm{~min}$ for resolution at $18 \mu \mathrm{m}$. The exposure time was $1,050 \mathrm{~ms}$, respectively, the voltage was $80 \mathrm{kV}$ in all cases, the current was $300 \mu \mathrm{A}$, and the rotation step $0.30^{\circ}$. The projection data were corrected for distortion and reconstructed by adjusting, smoothing and correction of ring artifact and beam hardening. The reconstructed isotropic voxel size was 9. Images were reconstructed with the scanner software (NRecon 1.6.6.0, Skyscan, Brucker micro-CT, Belgium) and converted to DICOM format for analysis. The time for reconstruction ranged from 2 to $60 \mathrm{~min}$, depending on the volume of the sample. Acquired images were rendered and exported as a three-dimensional (3D) volume. Post-processing analysis was performed using CTVox volume rendering 64 bit version, DATAVIEWER 64 bit version (Bruker, Kontich, Belgium), and Horos (v 2.4) software (free and open source code software at Horosproject.org).

\section{D Printing Protocol}

The micro-CT data served as the input for the $3 \mathrm{D}$ printing process. Images were imported in commercial software (Mimics v.21, Materialize, Leuven, Belgium) and reconstructed following steps of thresholding, region growing and semi-automatic image segmentation with manual editing where required, and the final volume meshes were exported as.stl files. All models were 1:1 in size as well as scaled with a 5-fold factor to provide more insight such small-sized cardiovascular structures. Each case was thus printed twice, in the two sizes. Prior to printing, models' surfaces were checked, and a scaffold was added (PreForm, Formlabs, ...). Models were printed in-house using a rigid white resin (Form2, Formlabs) and cleaned manually through standard steps of isopropanol washing and curing with final manual removal of the model's scaffold.

\section{RESULTS}

A total of $n=21$ cases were collected and reconstructed. Anamnestic data are summarized in Table 1. Samples consisted in heart $(19 / 21,90 \%)$ and heart-lungs $(2 / 21,10 \%)$. Mean gestational age at fetal echocardiography was $15.0 \pm 2.9$ weeks (range: 12-21 weeks). Mean gestational age at termination of pregnancy was $16.0 \pm 3.0$ weeks (range: $12-22$ weeks). Overall, $18 / 21(86 \%)$ cases suffered from cardiac anomalies, 15 (83\%) of which were complex CHD. 13/21 (62\%) cases had abnormal karyotype at invasive testing (chorionic villus sampling or amniocentesis). 10/21 (48\%) cases had associated extra-cardiac malformations (VACTERL syndrome, central nervous system anomalies including omphalocele and other anomalies of the posterior cranial fossa, cystic hygroma, cleft lips, renal anomalies including ptosis and polycystic kidneys, skeletal anomalies including anomalies of the arms, legs, vertebral column, and ribcage). Mean longitudinal diameter was $1.05 \pm 0.37 \mathrm{~cm}$ (range $0.4-1.7 \mathrm{~cm}$ ). Mean transverse diameter was $0.95 \pm 0.36 \mathrm{~cm}$ (range $0.4-1.8 \mathrm{~cm}$ ). Mean weight was $0.88 \pm 1.06 \mathrm{~g}$ (range: $0.39-4 \mathrm{~g}$ ).

Table 2 summarizes micro-CT acquisition properties.

All cases were successfully reconstructed, demonstrating the feasibility of the technique and of the protocols, including inhouse printing. Examples of 3D reconstructions are provided in Figure 1. Examples of 3D printed models are provided in Figure 2. When printed with the 5-fold magnification, models were also cut in a four-chamber view equivalent section, to provide insight into the intra-cardiac structures.

\section{DISCUSSION AND CONCLUSION}

Little is known about the feasibility of 3D printing from microCT datasets in the field of fetal cardiology. Traditionally, 3D printing of cardiovascular models relies on cardiac CT and MRI datasets of pediatric and adult human hearts in different settings according to established methodologies (12). Accuracy of postmortem micro-CT in ex-vivo evaluation of human fetal heart is validated. More recently, proof of principle experiences in $3 \mathrm{D}$ printing from micro-CT of human specimens in craniofacial surgery, placental imaging, archeological remains, bone imaging, 
TABLE 1 | Summary of cases included for 3D modeling, including main diagnosis, gestational age (GA) at diagnosis, and termination of pregnancy (TOP), karyotype, extra-cardiac anomalies, and dimensional assessment.

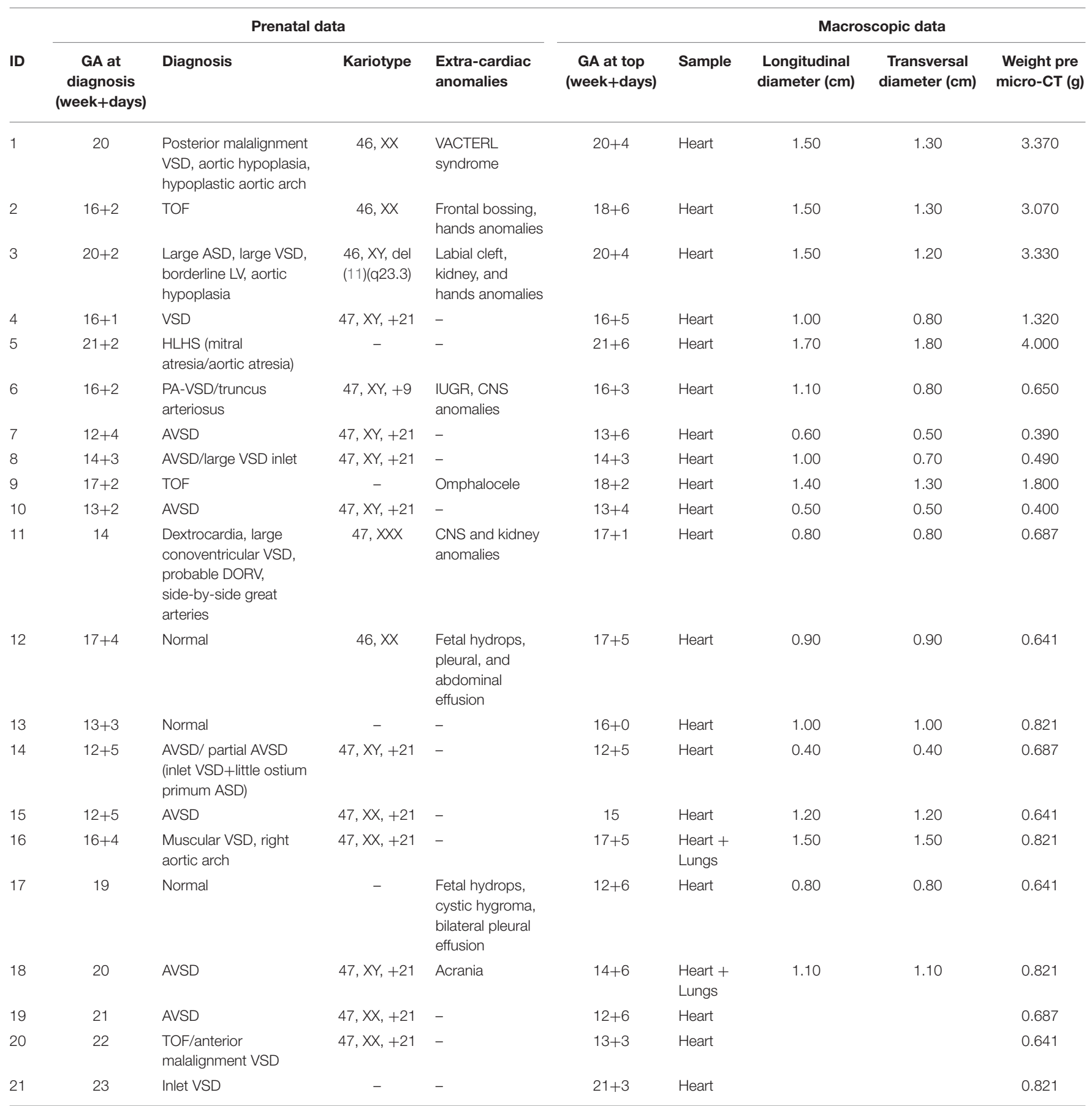

and cardiac anatomy has been presented (19). Shelmerdine et al. reported one example of 3D printing from micro-CT of an human fetal heart of 16 weeks of gestation, demonstrating that both main cardiac segments (atria, ventricles, and great vessels) and smaller component (cardiac valves, branching of the pulmonary artery and aorta, papillary muscle, and ventricular trabeculations) can be identified (19). There are any published data concerning earlier gestational age and larger case series. Our study complements these information on the feasibility of the technique and presents a case series comprehensive of complex cases of CHD, including hypoplastic left heart syndrome, double outlet right ventricle and tetralogy of Fallot from early and mid-gestational age (from 12 to 21 weeks of gestation). We agree with Shelmerdine's experience on feasibility and possible utility of 3D printing of human fetal heart from micro-CT. As compared to previous published projects, the novelty we 
TABLE 2 | Main settings for micro-CT acquisitions.

\begin{tabular}{|c|c|c|c|c|c|c|c|c|}
\hline ID & $\begin{array}{l}\text { Top to micro-CT } \\
\text { (days) }\end{array}$ & Lugol \% & Lugol (h) & Filter & $\begin{array}{l}\text { Resolution } \\
(\mu \mathrm{m})\end{array}$ & $\begin{array}{c}\text { Energy } \\
\text { range (V) }\end{array}$ & $\begin{array}{c}\text { Current } \\
\text { range }(\mu \mathrm{A})\end{array}$ & $\begin{array}{c}\text { Rotational } \\
\left.\text { step ( }{ }^{\circ}\right)\end{array}$ \\
\hline 1 & 68 & 25 & 72 & $\mathrm{Cu}+\mathrm{Al}$ & 18 & 500 & 89 & 264 \\
\hline 3 & 260 & 25 & 72 & $\mathrm{Cu}+\mathrm{Al}$ & 18 & 500 & 89 & 264 \\
\hline 4 & 178 & 20 & 72 & $\mathrm{Cu}+\mathrm{Al}$ & 18 & 500 & 89 & 264 \\
\hline 7 & 16 & 20 & 72 & Al 0,5 mm & 9 & 900 & 50 & 500 \\
\hline 8 & 16 & 20 & 72 & Al $0,5 \mathrm{~mm}$ & 18 & 210 & 50 & 500 \\
\hline 9 & 17 & 25 & 72 & $\mathrm{Cu}+\mathrm{Al}$ & 18 & 500 & 89 & 264 \\
\hline 10 & 16 & 20 & 72 & Al 0,5 mm & 9 & 900 & 50 & 500 \\
\hline 11 & 247 & 20 & 72 & $\mathrm{Cu}+\mathrm{Al}$ & 18 & 300 & 80 & 300 \\
\hline 16 & 74 & 30 & 72 & $\mathrm{Cu}+\mathrm{Al}$ & 18 & 300 & 80 & 300 \\
\hline 17 & 67 & 20 & 48 & Al $0,5 \mathrm{~mm}$ & 18 & 210 & 50 & 500 \\
\hline 18 & 66 & 30 & 72 & $\mathrm{Cu}+\mathrm{Al}$ & 18 & 300 & 80 & 300 \\
\hline 19 & 33 & 15 & 48 & Al 0,5 mm & 18 & 210 & 50 & 500 \\
\hline 20 & 41 & 30 & 72 & $\mathrm{Cu}+\mathrm{Al}$ & 18 & 300 & 80 & 300 \\
\hline 21 & 19 & 25 & 72 & $\mathrm{Cu}+\mathrm{Al}$ & 18 & 300 & 80 & 300 \\
\hline
\end{tabular}

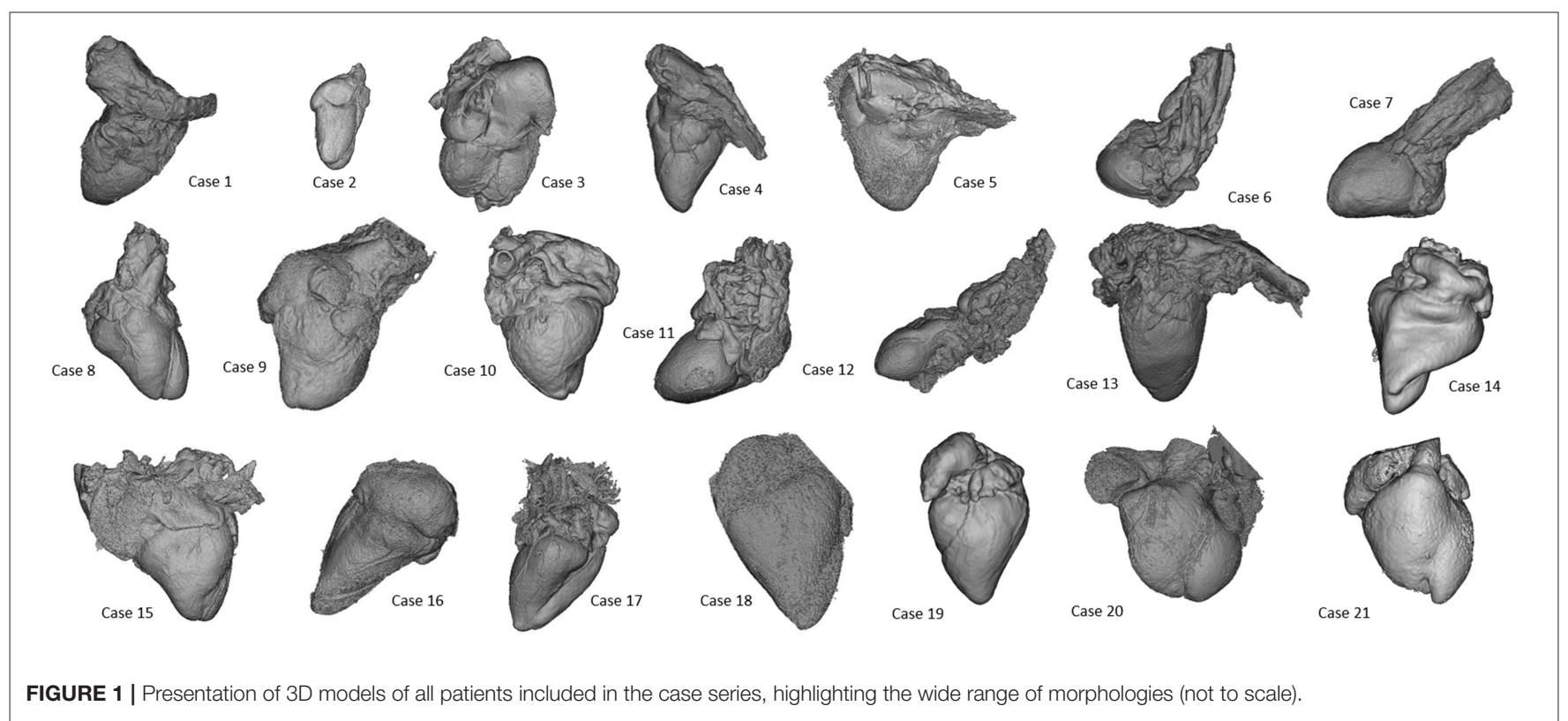

would like to demonstrate concerns the feasibility of microCT in a larger population of normal and pathological hearts with a wide range of gestational ages. The studied population consists of both normal and abnormal fetal hearts. Whilst the segmentation and printing processes are the same for normal and pathologic specimens, reconstruction of complex structures may require expert guidance in the identification of anatomical features of interest. Moreover, the population includes small samples (15/21 cases, $71 \%$ : weight $\leq 1 \mathrm{~g}$; $9 / 21$ cases, $43 \%$ : longitudinal and transverse diameter $\leq 1 \mathrm{~cm}$ ) and cases of low gestational age (10/21 cases, $48 \%$ : termination of pregnancy before the 15 weeks of gestation). This enabled us 


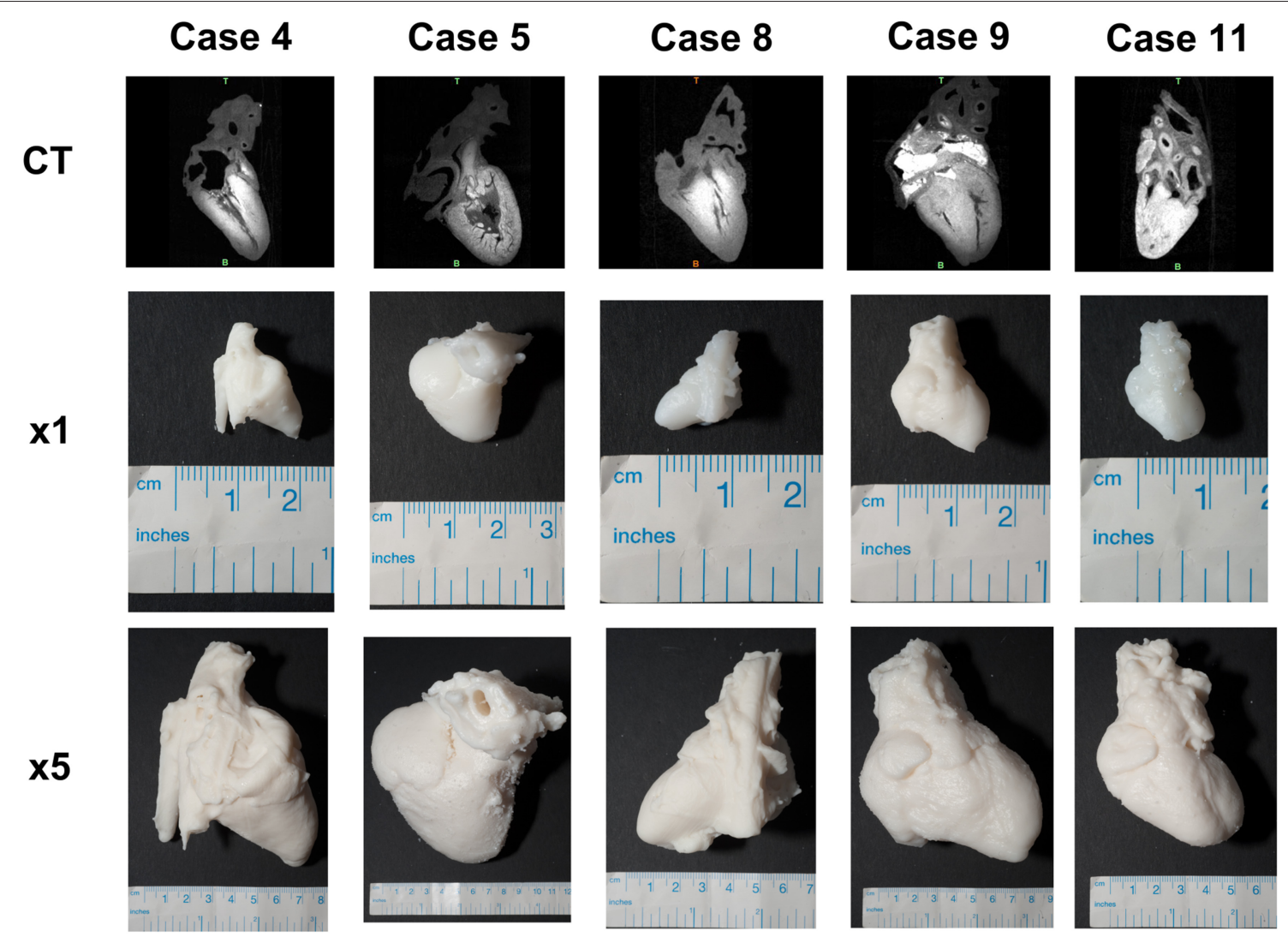

FIGURE 2 | Example of 3D printed models for a range of cases with different diagnoses, including the 1:1 3D printed model as well as the corresponding 5-fold scaled model for each case.

to demonstrate the feasibility of the process for both larger and smaller samples.

We focused only on technical aspects. Educational, research, and clinical implications remain to be addressed in the future and are beyond the scope of presenting this case series.

Virtual and printed models are nowadays used for didactic and research purposes in other areas of medicine, e.g., 3D printing fetal brains (22). These models can potentially represent a valuable resource for teaching fetal cardiac anatomy to medical students and trainees as well, presenting both normal fetal cardiovascular anatomy and congenital heart defects of different complexity. Whilst this should be systematically assessed in the context of cardiac morphology teaching, evidence is beginning to suggest that accurate $3 \mathrm{D}$ printed replicas can improve the teaching experience, reducing potential adverse reactions from students (e.g., in relation to cultural or religious backgrounds) and also holding the potential to show fetal development at different stages, to illustrate its full progression (21). Educational values of $3 \mathrm{D}$ printing models from micro-CT could play even a more important role in our era, where early fetal echocardiography is offered to high risk pregnancies since the eleventh week of gestation. The possibility to visualize small cardiac structure and to navigate into normal and pathological fetal heart offered by micro-CT could facilitate interpretation of echo images and, ultimately, improve medical knowledge. Moreover, integration of different imaging modalities also opens the door to research applications, which include 3D printing fetal heart models indeed derived from fetal echocardiographic data and integrated with 4D MRI flow information, which can also represent a valuable platform for in vitro modeling (18). Future application of $3 \mathrm{D}$ printed models could be related to familial counseling, fetal diagnosis, and more individualized planning of fetal and neonatal therapeutic strategies. On one hand, counseling applications may be limited due to the potentially confrontational and highly sensitive nature of the conversations and the role of the model as a communication tool in this context may potentially be counterproductive, as "reopening the wound" in revisiting the clinical conversations that ultimately led to terminating the pregnancy. On the other hand, 3D models (even scaled-up) could be used during familial counseling to facilitate familial awareness of fetal cardiac dimension and anatomy. Though biological effect of $\mathrm{X}$ ray radiation limits the in-vivo use of micro-CT and avoid patient-specific models, 3D reconstruction of first/early second trimester cardiac structure from micro-CT datasets is feasible. On the contrary, at present, data from $3 \mathrm{D}$ fetal echocardiography acquired during the first trimester has never been used to reconstruct and print fetal heart. 


\section{DATA AVAILABILITY STATEMENT}

The datasets generated for this study are available on request to the corresponding author.

\section{ETHICS STATEMENT}

Ethical review and approval was not required for the study on human participants in accordance with the local legislation and institutional requirements. The patients/participants provided their written informed consent to participate in this study. Written informed consent was obtained from the individual(s) for the publication of any potentially identifiable images or data included in this article.

\section{REFERENCES}

1. Donofrio MT, Moon-Grady AJ, Hornberger LK, Copel JA, Sklansky MS, Abuhamad A, et al. Diagnosis and treatment of fetal cardiac disease: a scientific statement from the American Heart Association. Circulation. (2014) 129:2183-242. doi: 10.1161/01.cir.0000437597.44550.5d

2. Hutchinson D, McBrien A, Howley L, Yamamoto Y, Sekar P, Motan T, et al. First-trimester fetal echocardiography: identification of cardiac structures for screening from 6 to 13 weeks' gestational age. J Am Soc Echocardiogr. (2017) 30:763-72. doi: 10.1016/j.echo.2017.03.017

3. Rossi AC, Prefumo F. Correlation between fetal autopsy and prenatal diagnosis by ultrasound: a systematic review. Eur J Obstet Gynecol Reprod Biol. (2016) 210:201-6. doi: 10.1016/j.ejogrb.2016.12.024

4. Dickinson JE, Prime DK, Charles AK. The role of autopsy following pregnancy termination for fetal abnormality. Aust N Z J Obstet Gynaecol. (2007) 47:445-9. doi: 10.1111/j.1479-828X.2007.00777.x

5. Taylor AM, Arthurs OJ, Sebire NJ. Postmortem cardiac imaging in fetuses and children. Pediatr Radiol. (2015) 45:549-55. doi: 10.1007/s00247-014-3164-0

6. Degenhardt K, Wright AC, Horng D, Padmanabhan A, Epstein JA. Rapid 3D phenotyping of cardiovascular development in mouse embryos by micro-CT with iodine staining. Circ Cardiovasc Imaging. (2010) 3:31422. doi: 10.1161/CIRCIMAGING.109.918482

7. Lombardi CM, Zambelli V, Botta G, Moltrasio F, Cattoretti G, Lucchini V, et al. Postmortem microcomputed tomography (micro-CT) of small fetuses and hearts. Ultrasound Obstet Gynecol. (2014) 44:600-9. doi: 10.1002/uog.13330

8. Hutchinson JC, Arthurs OJ, Ashworth MT, Ramsey AT, Mifsud W, Lombardi $\mathrm{CM}$, et al. Clinical utility of postmortem microcomputed tomography of the fetal heart: diagnostic imaging vs. macroscopic dissection. Ultrasound Obstet Gynecol. (2016) 47:58-64. doi: 10.1002/uog.15764

9. Votino C, Jani J, Verhoye M, Bessieres B, Fierens Y, Segers V, et al. Postmortem examination of human fetal hearts at or below 20 weeks' gestation: a comparison of high field MRI at $9.4 \mathrm{~T}$ with lower-field MRI magnets and stereomicroscopic autopsy. Ultrasound Obstet Gynecol. (2012) 40:437-44. doi: 10.1002/uog.11191

10. Sandaite I, Dymarkowski S, De Catte L, Moerman P, Gewillig M, Fedele L, et al. Fetal heart pathology on postmortem 3-T magnetic resonance imaging. Prenat Diagn. (2014) 34:223-9. doi: 10.1002/pd.4283

11. Taylor AM, Sebire NJ, Ashworth MT, Schievano S, Scott RJ, Wade A, et al. Postmortem cardiovascular magnetic resonance imaging in fetuses and children: a masked comparison study with conventional autopsy. Circulation. (2014) 129:1937-44. doi: 10.1161/CIRCULATIONAHA.113.005641

12. Vukicevica M, Mosadeghb B, Minb JK, Littlea SH. Cardiac 3D printing and its future directions. JACC Cardiovasc Imaging. (2017) 10: 17184. doi: 10.1016/j.jcmg.2016.12.001

13. Batteu C, Haidar MA, Bonnet D. 3d-printed models for surgical planning in complex congenital heart diseases: a systematic review. Front Pediatr. (2019) 7:23. doi: 10.3389/fped.2019.00023

\section{AUTHOR CONTRIBUTIONS}

CS: conception and design of study, analysis and interpretation of data, and drafting of manuscript. CL: conception and design of study, drafting of manuscript, and final approval. AS and MO: analysis and interpretation of data. MC, FP, GL, and LR: final approval. GB: conception and design of study, analysis and interpretation of data, drafting of manuscript, and final approval.

\section{ACKNOWLEDGMENTS}

The authors acknowledge the generous support of the Grand Appeal (Bristol Children's Hospital Charity) and the British Heart Foundation.

14. Valverde I, Gomez-Ciriza G, Hussain T, Suarez-Mejias C, Velasco-Forte MN, Byrne $\mathrm{N}$, et al. Three-dimensional printed models for surgical planning of complex congenital heart defects: an international multicentre study. Eur J Cardiothorac Surg. (2017) 52:1139-48. doi: 10.1093/ejcts/ezx208

15. Kiraly L, Kiraly B, Szigeti K, Tamas CZ, Daranyi S. Virtual museum of congenital heart defects: digitization and establishment of a database for cardiac specimens. Quant Imaging Med Surg. (2019)1:115-26. doi: 10.21037/qims.2018.12.05

16. Chen SA, Ong CS, Hibino N, Baschat AA, Garcia JR, Miller JL. 3D printing of fetal heart using 3D ultrasound imaging data. Ultrasound Obstet Gynecol. (2018) 52:808-9. doi: 10.1002/uog.19166

17. Chambers Gurson S. Advances in fetal echocardiography: myocardial deformation analysis, cardiac MRI and three-dimensional printing. Curr Opin Cardiol. (2019) 34:35-40. doi: 10.1097/HCO.00000000000 00584

18. Ruedinger KL, Zhou $\mathrm{H}$, Trampe B, Heiser $\mathrm{T}$, Srinivasan $\mathrm{S}$, Iruretagoyena JI, et al. Modeling fetal cardiac anomalies from prenatal echocardiography with 3-dimensional printing and 4-dimensional flow magnetic resonance imaging. Circ Cardiovasc Imaging. (2018) 11:e007705. doi: 10.1161/CIRCIMAGING.118.007705

19. Shelmerdine SC, Simcock IC, Hutchinson JC, Aughwane R, Melbourne A, Nikitichev DI, et al. 3D printing from microfocus computed tomography (micro-CT) in human specimens: education and future implications. $\mathrm{Br} \mathrm{J}$ Radiol. (2018) 91:20180306. doi: 10.1259/bjr.20180306

20. Sandrini C, Rossetti L, Zambelli V, Zanarotti R, Bettinazzi F, Soldà $\mathrm{R}$, et al. Accuracy of micro-computed tomography in post mortem evaluation of fetal congenital heart disease. Comparison between postmortem micro-ct and conventional autopsy. Front Pediatr. (2019) 7:92. doi: 10.3389/fped.2019.00092

21. Young JC, Quayle MR, Adams JW, Bertram JF, McMenamin PG. Threedimensional printing of archived human fetal material for teaching purposes. Anat Sci Educ. (2019) 1:90-6. doi: 10.1002/ase.1805

22. Jarvis D, Griffiths PD, Majewski C. Demonstration of normal and abnormal fetal brains using 3D printing from in utero MR imaging data. AJNR Am J Neuroradiol. (2016) 37:1757-61. doi: 10.3174/ajnr.A4783

Conflict of Interest: The authors declare that the research was conducted in the absence of any commercial or financial relationships that could be construed as a potential conflict of interest.

Copyright (C) 2020 Sandrini, Lombardi, Shearn, Ordonez, Caputo, Presti, Luciani, Rossetti and Biglino. This is an open-access article distributed under the terms of the Creative Commons Attribution License (CC BY). The use, distribution or reproduction in other forums is permitted, provided the original author $(s)$ and the copyright owner(s) are credited and that the original publication in this journal is cited, in accordance with accepted academic practice. No use, distribution or reproduction is permitted which does not comply with these terms. 